\title{
Actividad electroencefalográfica durante tareas de simulación de conducción con videojuegos
}

\author{
Sebastián-Guerrero M.V. ${ }^{1}$, Arcos-Sánchez C. ${ }^{2}$, Navascués-Sanagustín M.A. ${ }^{3}$, Idiazábal-Alecha M.A. ${ }^{4}$, \\ Ruiz-López C. ${ }^{5}$, Arana-Aritméndiz M.V. ${ }^{6}$
}

Sanid. mil. 2021; 77 (2): 91-89, ISSN: 1887-8571

Este trabajo ha sido financiado por los Proyectos (CUD-ID: 2018-02, 2019-04), del Centro Universitario de la Defensa-Academia General Militar, Zaragoza, España.

\section{RESUMEN}

Antecedentes y Objetivos. El estudio de la función atencional, foco de interés en el campo de las neurociencias, puede realizarse mediante la cuantificación electroencefalográfica, técnica no invasiva que permite evaluar la actividad cerebral. El objetivo de este trabajo es estudiar la atención/concentración del personal de las Fuerzas Armadas españolas durante la simulación de manejo de vehículos militares. Se analizan para ello las variaciones en la complejidad numérica bioeléctrica cortical al pasar de estados basales a tareas de simulación, y con la dificultad de dichas tareas. Material y métodos. Se han analizado las señales electroencefalográficas de 36 sujetos voluntarios con la Movilidad, estimador de la frecuencia media de la señal que mide la desviación estándar de la derivada de la señal en referencia a la desviación estándar de la amplitud. Los datos se han estudiado estadísticamente mediante: análisis exploratorio, análisis de varianza de dos factores y prueba de hipótesis. Resultados. Se observa un aumento del promedio cortical de la Movilidad en cada tipo de electroencefalograma a medida que la tarea se vuelve más compleja y requiere más atención, y una disminución a medida que cesa la actividad. La Movilidad muestra un aumento considerable en el área occipital y parte de las áreas temporales durante las simulaciones, lo que indica la activación de las redes del área visual primaria y la corteza auditiva primaria. Conclusiones. La Movilidad detecta cambios en la actividad de diferentes áreas corticales asociados a las tareas cognitivas, por lo que puede considerarse un buen indicador de la actividad cerebral.

PALABRAS CLAVE. Actividad cerebral. Atención. Cuantificación. Electroencefalograma. Movilidad. Simulación de conducción militar.

\section{Electroencephalographic activity during tasks of driving simulation with videogames \\ SUMMARY}

Background and Objectives. The study of the attentional function, a focus of interest in the neurosciences field, can be carried out through electroencephalographic quantification, a non-invasive technique that enables the evaluation of the brain activity. The objective of this work is to analyze the attention/concentration of staff of the Spanish Armed Forces in tactical simulations of handling military vehicles. For this purpose, variations in cortical bioelectric numerical complexity are analyzed when moving from resting states to simulation tasks, and with the difficulty of the task. Material and methods. Electroencephalographic signals were analyzed from a group of 36 volunteer subjects with the Mobility, an estimator of the average frequency of the signal that measures the standard deviation of the derivative of the signal in reference to the standard deviation of the amplitude. The data have been statistically studied by means of an exploratory survey, an analysis of variance of two factors and a hypothesis test. Results. An increase of the cortical average of Mobility is observed in each type of electroencephalogram as the task becomes more complex and requires more attention, and decreases as the activity ceases. Mobility shows a considerable increase in the occipital and part of the temporal areas during the simulations, which shows the activation of the signals corresponding to the primary visual area and the primary auditory cortex.

Conclusions. Mobility detects changes in the activity of different cortical areas with the change in cognitive tasks. Thus, it can be considered a good indicator of brain activity.

KEY WORDS: Attention. Brain activity. Electroencephalogram. Military driving simulation. Mobility. Quantification.

1. Centro Universitario de la Defensa-Academia General Militar. Zaragoza

2. Comandante Médico. Servicio de Neurología. Hospital General de la Defensa. Zaragoza

3. Universidad de Zaragoza

4. Instituto Neurocognitivo Incia.Barcelona.

5. Teniente Coronel. Centro Universitario de la Defensa. Academia General Militar. Zaragoza.

6. Servicio de Neurología. Hospital General de la Defensa. Zaragoza.

Dirección para correspondencia: María Victoria Sebastián Guerrero. Centro Universitario de la Defensa-Academia General Militar. Ctra. Huesca s/n, 50090 Zaragoza, Spain.msebasti@unizar.es.Tfno: 976739851; FAX: 976739824

Recibido: 04 de febrero de 2020

Aceptado: 23 de febrero de 2021

doi: 10.4321/S1887-85712021000200003

\section{INTRODUCCIÓN}

El estudio de la función atencional es un foco de interés en el campo de las neurociencias, principalmente en relación con el aumento de las técnicas y metodologías no invasivas, que permiten la evaluación de la actividad cerebral con resolución espacial y temporal, revelando las estructuras involucradas en cada proceso sensorial, perceptivo y cognitivo.

Posner ${ }^{1}$, inicialmente declaró que las manifestaciones atencionales son producto de sistemas o estructuras cerebrales separadas, aunque están interrelacionadas. Bajo esta conceptualización, la atención se entendería como un sistema modular 
compuesto por tres redes: la red de atención posterior u orientación, la red de vigilancia o advertencia y la red anterior o control ejecutivo. Cada una de estas redes sería responsable de diferentes funciones de atención y, a su vez, se asociaría con diferentes áreas del cerebro en un trabajo conjunto que también considera la individualidad del sujeto, incorporando aspectos evolutivos, psicosociales y emocionales ${ }^{2}$. La atención puede considerarse la base fundamental para el desarrollo eficiente de los procesos cognitivos y el control de la conducta.

Colmenero et al. ${ }^{3}$ definen la atención como un mecanismo central de control del procesamiento de la información, que actúa de acuerdo con los objetivos del organismo mediante la activación e inhibición de procesos. También puede orientarse hacia los sentidos, las estructuras del conocimiento en la memoria y los sistemas de respuesta.

El primer requisito para las personas que realizan tareas que pueden ser monótonas es la vigilancia mantenida o la atención sostenida. Así, el estudio de la atención y la pérdida de la misma durante la realización de diferentes tareas es un tema de interés actual desde el punto de vista neurológico ${ }^{4}$, ya que constituye el requisito previo para otros procesos cognitivos complejos. El estudio de la atención durante la ejecución de diversas tareas mentales se vuelve más importante cuando se analiza durante el desempeño de tareas por parte del personal militar ${ }^{5}$, en el sector de la salud o en la industria o en aquellos casos en que la disminución de la vigilancia podría tener consecuencias peligrosas tales como accidentes, pérdida de vidas, etc.

La conducción de vehículos militares requiere una especial atención/concentración, ya que se realiza a la vez que otras tareas tácticas militares ${ }^{6}$, como monitorear las comunicaciones o discriminar posibles objetivos enemigos ${ }^{7}$. Todo esto unido a la orografía del terreno en el que se lleva a cabo la conducción, no siempre fácil, hace que los conductores militares tengan que lidiar con situaciones que requieren de máxima concentración y se vean sometidos a una posible sobrecarga atencional ${ }^{8,9}$.

Lu et a ${ }^{10}$ analizan la carga de trabajo durante la conducción de un vehículo de ruedas multipropósito de alta movilidad simulado mientras se realiza una tarea de memoria. La sobrecarga de la tripulación de un carro de combate en escenarios típicos de conflicto, dotado de nuevas tecnologías, ha sido estudiada por Mao et $\mathrm{al}^{11}$.

El estudio de la actividad bioeléctrica cortical mediante el electroencefalograma (EEG), permite analizar las sobrecarga, atención y concentración requeridas durante la realización de tareas de conducción o simulación de estas. Los dispositivos modernos de electroencefalografía, más ligeros y portátiles, permiten la recopilación de registros EEG en todo tipo de escenarios. Para el análisis del EEG se pueden utilizar algunas de las técnicas con representaciones de frecuencia de tiempo, como la Transformada rápida de Fourier o la Transformada de Fourier de tiempo reducido, que muestran la evolución temporal de las características espectrales de la señal. El análisis del EEG puede ampliarse mediante la aplicación de cuantificadores de la dinámica no lineal, técnicas fractales y nuevos métodos espectrales ${ }^{12}$. Por ejemplo, la dimensión de correlación ha sido utilizada por Yang et al..$^{13}$ para medir los cambios en la actividad cerebral en el registro de varios tipos de EEG.
Nuestro grupo ha propuesto métodos analíticos y computacionales avanzados para obtener cuantificadores del EEG (de tipo espectral y fractal) que asignan valores numéricos al sistema observado y permiten establecer comparaciones entre diferentes EEG y diferenciar los estados cerebrales correspondientes a los procesos mentales. Estos han sido aplicados al estudio de la atención en niños con trastorno de déficit de atención e hiperactividad (TDAH) durante la realización de diferentes tareas ${ }^{14-15}$.

El neurofisiólogo B. Hjorth en 1970 definió un conjunto de parámetros utilizados como herramienta clínica en la descripción cuantitativa del $\mathrm{EEG}^{16}$, denominados: Actividad, Movilidad y Complejidad. Estos cuantificadores se conocen como parámetros de Hjorth o descriptores de pendiente normalizados, y describen una señal en el dominio del tiempo y la frecuencia .

En este trabajo se utiliza la Movilidad con el objetivo de estudiar las variaciones en la complejidad numérica bioeléctrica cortical de un grupo de sujetos de las Fuerzas Armadas al pasar de estados de reposo a tareas de simulación de conducción de vehículos militares, y con la dificultad de la tarea. La Movilidad se considera una medida de la desviación estándar de la derivada de la señal en referencia a la desviación estándar de la amplitud. Es un estimador de la frecuencia media de la señal que describe de manera global el contenido espectral del registro, aumentando cuando la frecuencia media aumenta, y disminuyendo en otro caso. Se ha demostrado en trabajos anteriores, que es un buen indicador de la actividad cerebral, variando en las áreas involucradas en el desempeño de diversas tareas de simulación ${ }^{15,17}$.

\section{MATERIAL Y MÉTODOS}

El estudio está en concordancia con la Ley de Investigación Biomédica (Ley 14/2007, de 3 de julio de investigación biomédica) y con la Declaración de Helsinki. El protocolo de investigación y el consentimiento informado fueron aprobados por el Comité Ético de Investigación Clínica de la Inspección General de Salud de la Defensa. Cada participante firmó su correspondiente hoja de información del paciente y el consentimiento informado antes de las pruebas.

\section{Sujetos y diseño experimental}

Se solicitó la participación voluntaria de 40 sujetos entre los Caballeros y Damas Cadetes alumnos de primer curso de la Academia General Militar, y de Oficiales y Suboficiales de dicha Academia y del Hospital General de la Defensa de Zaragoza. La muestra incluyó individuos diestros sin antecedentes neurológicos y que no tomaban medicación crónica, sin antecedentes de daño cerebral, problemas de visión o dificultades de aprendizaje. Se seleccionaron 36 sujetos (25 hombres y 11 mujeres) cuyas señales EEG estaban libres de artefactos. La edad media del grupo fue de $31.44( \pm 13.05)$.

Las señales EEG analizadas fueron recogidas en el Hospital General de la Defensa de Zaragoza. Para cada sujeto, se registraron 6 tipos de señales EEG de 3 minutos de duración en reposo y 


\section{Actividad electroencefalográfica durante tareas de simulación de conducción con videojuegos}

durante la ejecución de varios ejercicios tácticos. Las secuencias se realizaron siempre en el orden que se indica a continuación:

- (oc): Reposo con ojos cerrados.

- (oa): Reposo con ojos abiertos.

- (j1): Simulación del manejo de un vehículo de combate con un videojuego.

- (j2): Realización de la tarea (j1) mientras el sujeto recibe estímulos distractores externos.

- (j3): Repetición del ejercicio de simulación (j1) sin estímulos externos.

- (oa2): Reposo con ojos abiertos tras finalizar la tarea (j3).

La simulación de conducción del vehículo militar se realizó en un Ipad con la aplicación Tank Ace. El sujeto debía prestar atención a la conducción en un terreno de orografía complicada (tipo Afganistán), a los ataques de vehículos enemigos y a los parámetros del vehículo.

En la tarea (j2), el sujeto simultaneaba la tarea (j1) con la respuesta de una serie de preguntas no relacionadas con la prueba (ver Fig. 1).

Las pruebas se realizaron en un recinto débilmente iluminado, silencioso, a temperatura constante y en una sala eléctricamente blindada, siempre entre las 10 y las 14 horas.

Las señales EEG de cada sujeto se grabaron con un equipo ambulatorio digital con el software Compumedics Limited Profusion EEG 4, montaje monopolar (con electrodos de Ag/ $\mathrm{AgCl}$ ) y 16 canales del Sistema 10-20 internacional de Jasper referenciados a $\mathrm{Cz}$, de los que se analizaron 10: F7, F8, O1, O2, P3, P4, T3, T4, T5 y T6. Se aplicaron filtros con frecuencias de corte $0.5 \mathrm{~Hz}$ y $70 \mathrm{~Hz}$. Una vez registrada la señal, se digitalizó con un conversor muestreando a 256 puntos por segundo. Tras una inspección visual de las señales se decidió analizar el tercer minuto de cada una de las señales por ser el segmento que, en general, presentaba el menor número de artefactos.

Antes de la realización de las pruebas electroencefalográficas los sujetos respondieron al cuestionario de salud del ejército (Anexo a la Instrucción 22/2008 de 31 de enero) y a varios cuestionarios con información personal.

\section{Cuantificación EEG}

La Movilidad (), parámetro de cuantificación analizado en este trabajo, describe una señal en el dominio del tiempo, (amplitud, pendiente, relación de cambios de pendiente) y la frecuencia (Transformada de Fourier). Se calcula a partir de los momentos espectrales () de la señal electroencefalográfica:

$$
\begin{gathered}
\mathcal{M}=\left(m_{2} / m_{0}\right)^{1 / 2} \\
m_{n}=\frac{1}{2 \pi} \int_{-\infty}^{+\infty} w^{n} S(w) d w
\end{gathered}
$$

donde , el complejo conjugado de y su módulo. Se ha deducido la fórmula de en función de los coeficientes espectrales ${ }^{15}$,

$$
\mathcal{M}=\left(\frac{m_{2}}{m_{0}}\right)^{1 / 2}=\frac{\omega_{0}\left(\sum_{m=1}^{\infty} m^{2}\left(c_{m}^{2}+d_{m}^{2}\right)\right)^{1 / 2}}{\left(2 c_{0}^{2}+\sum_{m=1}^{\infty}\left(c_{m}^{2}+d_{m}^{2}\right)\right)^{1 / 2}}
$$

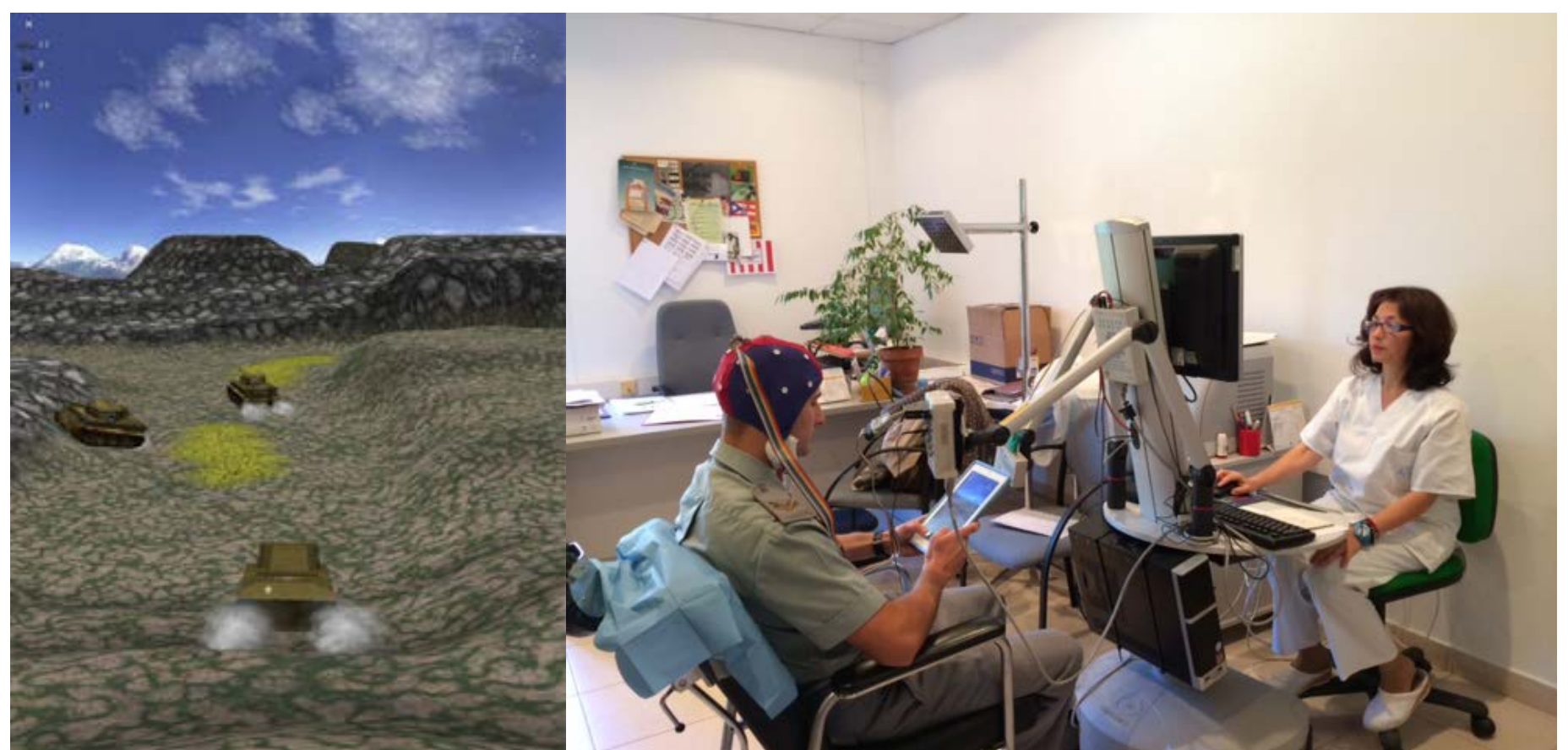

Figura 1. La imagen de la derecha recrea la simulación de conducción de un vehículo de combate con un videojuego en un escenario de orografía complicada (tipo Afganistán). En la imagen de la derecha se observa un sujeto en el Servicio de Neurología del Hospital General de la Defensa durante la realización de la simulación con los electrodos en su cuero cabelludo y la DUE encargada de recoger las señales EEG. 
Tabla 1. Valores promedio \pm desviaciones típicas de la Movilidad en cada canal (F7, F8, O1, O2, P3, P4, T3, T4, T5, T6) para cada tipo de EEG, estados basales con ojos cerrados (oc) y abiertos antes y después de las simulaciones (oa y oa2) y tareas de simulación de conducción de vehículos militares (j1, j2 y j3), (n=36). La última línea muestra el promedio cortical de los diez canales para cada tipo de EEG.

\begin{tabular}{|c|c|c|c|c|c|c|c|}
\hline & & Basal oc & Basal oa (pre) & Tarea j1 & Tarea j2 & Tarea j3 & Basal oa2 (post) \\
\hline \multirow{11}{*}{ 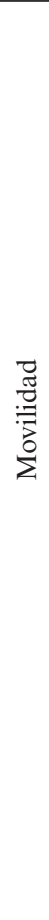 } & F7 & $82,928 \pm 23,128$ & $90,415 \pm 21,507$ & $71,567 \pm 17,417$ & $73,639 \pm 14,861$ & $70,693 \pm 15,369$ & $90,223 \pm 18,736$ \\
\hline & F8 & $83,270 \pm 19,736$ & $90,012 \pm 21,847$ & $71,094 \pm 16,862$ & $78,525 \pm 18,503$ & $71,575 \pm 17,439$ & $91,150 \pm 24,668$ \\
\hline & $\mathrm{O} 1$ & $76,432 \pm 14,532$ & $78,093 \pm 18,195$ & $93,522 \pm 27,127$ & $92,322 \pm 27,478$ & $89,556 \pm 26,503$ & $76,188 \pm 17,483$ \\
\hline & $\mathrm{O} 2$ & $76,719 \pm 17,749$ & $75,671 \pm 16,619$ & $93,170 \pm 26,880$ & $92,868 \pm 27,999$ & $93,684 \pm 32,331$ & $75,795 \pm 16,590$ \\
\hline & P3 & $82,946 \pm 20,858$ & $81,874 \pm 22,490$ & $82,507 \pm 21,338$ & $81,450 \pm 19,437$ & $84,043 \pm 23,169$ & $77,047 \pm 17,006$ \\
\hline & P4 & $83,511 \pm 20,828$ & $82,207 \pm 19,664$ & $82,962 \pm 17,482$ & $81,557 \pm 17,200$ & $82,488 \pm 18,906$ & $78,776 \pm 17,034$ \\
\hline & T3 & $92,978 \pm 22,829$ & $97,668 \pm 23,850$ & $87,972 \pm 20,883$ & $96,734 \pm 22,158$ & $87,595 \pm 19,270$ & $96,129 \pm 20,612$ \\
\hline & $\mathrm{T} 4$ & $90,045 \pm 21,769$ & $90,047 \pm 21,361$ & $91,412 \pm 24,638$ & $98,351 \pm 25,620$ & $90,821 \pm 22,359$ & $92,371 \pm 22,204$ \\
\hline & T5 & $77,180 \pm 13,170$ & $79,458 \pm 17,876$ & $92,244 \pm 23,047$ & $87,058 \pm 14,832$ & $95,923 \pm 23,105$ & $79,271 \pm 15,326$ \\
\hline & T6 & $76,498 \pm 15,102$ & $79,916 \pm 21,863$ & $94,528 \pm 21,830$ & $96,260 \pm 17,511$ & $93,540 \pm 21,330$ & $79,353 \pm 21,306$ \\
\hline & $\begin{array}{l}\text { Promedio } \\
\text { Cortical }\end{array}$ & 82,251 & 84,536 & 86,098 & 87,876 & 85,992 & 83,630 \\
\hline
\end{tabular}

\section{Análisis Estadístico}

Tras obtener los valores de la Movilidad de Hjorth para cada uno de los 10 canales y 6 tipos de EEG analizados, se realizó un estudio estadístico completo de los resultados obtenidos.

Se llevó a cabo un análisis exploratorio de los datos acompañado de diagramas de perfil y gráficos Box-Plot, seguido de un ANOVA de dos factores intra-sujeto con medidas repetidas en ambos factores (canal con 10 niveles y tipo de EEG con 6 niveles). Se utilizó el corrector épsilon de Greenhouse-Geisser ante la violación del supuesto de esfericidad. Se hicieron comparaciones múltiples por pares de los efectos principales, tanto para el factor canal como para el factor tarea. Los niveles críticos y los intervalos de confianza se ajustaron mediante la corrección de Bonferroni.

Para detectar la existencia de diferencias significativas entre los tres primeros tipos de EEG (oc, oa, j1) en cada canal, tomados por pares, se aplicó una prueba de hipótesis de igualdad de medias. El proceso se repitió para los tres tipos de EEG en las simulaciones militares $(\mathrm{j} 1, \mathrm{j} 2, \mathrm{j} 3)$ y para los dos tipos de EEG basales con los ojos abiertos (oa, oa2).

En cada caso se aplicó la prueba de Kolmogorov-Smirnov para comprobar la hipótesis de normalidad de los datos y la prueba de Levene para la hipótesis de homocedasticidad (igualdad de varianzas). No se rechazaron las hipótesis anteriores en ningún canal, por lo que se aplicó una prueba paramétrica $T$ para muestras relacionadas que permitió contrastar el supuesto de que la diferencia de medias entre los dos tipos de EEG era cero en cualquiera de los 10 electrodos con un nivel de confianza del 95\% ().

\section{RESULTADOS}

\section{Análisis Exploratorio}

Las medias de los datos obtenidos en la Movilidad de Hjorth para cada tipo de EEG y en cada electrodo se muestran en la Tabla 1, cuya última fila representa el promedio cortical del cuantificador. Se puede observar un aumento de este promedio en cada tipo de EEG a medida que la tarea se vuelve más compleja y requiere mayor atención, y disminuye a medida que cesa la actividad.

Analizando los promedios para la Movilidad en cada tipo de EEG y cada electrodo o canal, se observa que los valores del cuantificador varían en el intervalo (70-99). El valor mínimo registrado se da en el electrodo F7 de la zona frontal en la tarea de simulación (j3) y el máximo en el área temporal (T4) en la tarea $(\mathrm{j} 2)$, que requiere la mayor atención.

Durante los ejercicios de simulación de vehículos militares (j1), (j2) y (j3), los valores máximos del parámetro se registran en las áreas occipital y temporal, mientras que los valores mínimos se localizan en la zona frontal (ver Fig.2).

Al comparar los tipos de EEG, se observa que la Movilidad aumenta (y, por lo tanto, la frecuencia) en el área occipital, al pasar de los estados basales al desempeño de las tareas de simulación (j1), (j2) y (j3) (ver Fig. 3a). Este aumento también se refleja en parte del área temporal (T4, T5 y T6) (ver Fig. 3b). Esto se explica por la activación del área visual primaria y el 


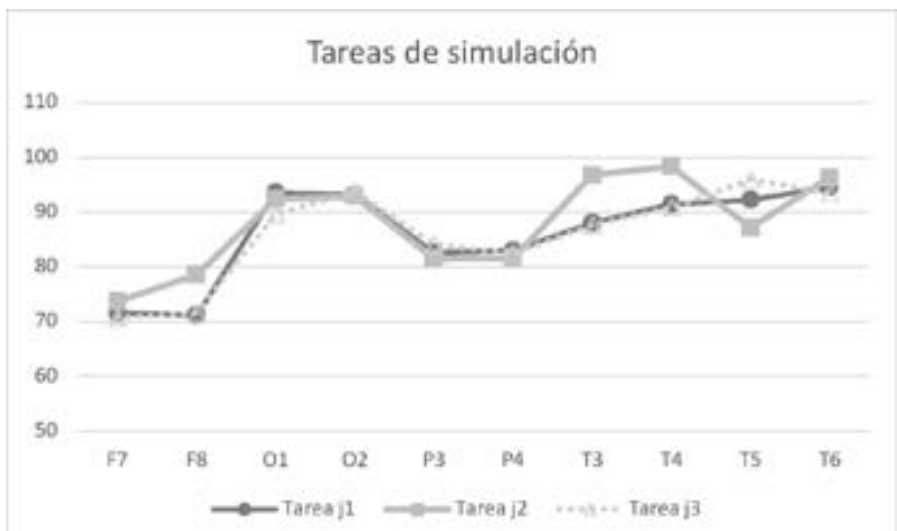

Figura 2. Valores promedio de la Movilidad en cada canal (F7, F8, O1, O2, P3, P4, T3, T4, T5, T6) para cada una de las tareas de simulación de conducción de vehículos militares (j1, j2 y j3). Se observa un aumento de la Movilidad en la zona occipital (canales O1 y O2) en todas las tareas, así como en la zona temporal (T3 y T4) en la tarea j2 (con estímulos distractores externos).

área temporal del cerebro (corteza auditiva primaria) durante la realización de la tarea de simulación. Ambas áreas se involucran en este tipo de tareas (el videojuego está equipado con sonido).

Los gráficos Box-Plot permiten visualizar el comportamiento de la Movilidad en el área occipital en diferentes tipos de EEG (basal con los ojos cerrados (oc), tarea de simulación (j1), basal con los ojos abiertos (oa)) (ver Fig. 4).

Se puede observar cómo los aumentos y disminuciones del parámetro no ocurren de manera uniforme en todas las áreas del cerebro. El cambio del estado de reposo con los ojos cerrados (oc) al reposo con los ojos abiertos (oa) produce un ligero aumento en el parámetro en las áreas frontal y temporal. El inicio de la simulación de conducción del vehículo militar tras el estado de reposo con los ojos abiertos se refleja con un marcado aumento de la Movilidad en el área occipital $(\mathrm{O} 1, \mathrm{O} 2)$ y en parte del área temporal (T5, T6). La disminución mayor ocurre en el área frontal (F7, F8) (ver Fig. 5a).

Después de la simulación (j3), una vez que el juego ha finalizado y el individuo vuelve a un estado basal con los ojos abiertos, hay un aumento considerable del parámetro en el área frontal (F7, F8) y una disminución notable en las áreas occipital y temporal (O1, O2, T5, T6) (ver Fig. 5b).

\section{Análisis de Varianza}

Los resultados obtenidos en el análisis de varianza (ANOVA) muestran el estadístico univariante $\mathrm{F}$ asociado a cada factor y su p-valor. Para el factor canal se obtuvieron diferencias significativas $\left(F(4,992)=8,067, p=710^{-7}\right)$. En el caso del factor tarea no se encontraron diferencias significativas $(\mathrm{F}(3,749)=0,795, \mathrm{p}=$ 0,523). La interacción canal-tarea también presentó diferencias significativas entre sus niveles $\left(\mathrm{F}(13,781)=13,545, \mathrm{p}=610^{-27}\right)$.

\section{Test de Hipótesis}

En la última fase del estudio estadístico, se aplicó la prueba paramétrica $T$ para muestras relacionadas. La Tabla 2 muestra los resultados de los $p$-valores para aquellas comparaciones en las que se encontraron diferencias significativas.

Al analizar los resultados obtenidos con el contraste $T$, se puede observar que no hay diferencias significativas entre el EEG en reposo con ojos cerrados y con ojos abiertos en ninguno de los electrodos estudiados. Las diferencias significativas entre el EEG en reposo con ojos cerrados y la tarea de simulación (j1) se dan en las áreas occipital y temporal (canales O1, O2, T5 y
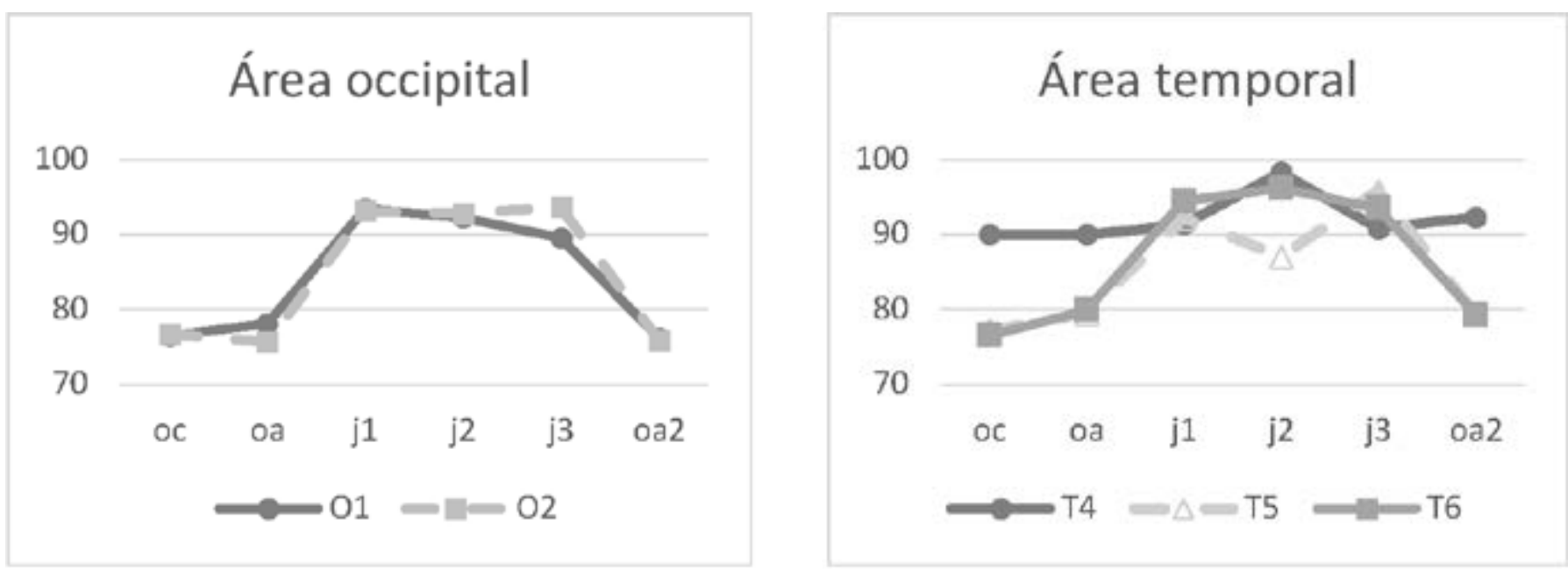

Figura 3. Valores promedio de la Movilidad en cada uno de los tipos de EEG, basal con ojos cerrados (oc), estado basal con ojos abiertos (oa), tareas de simulación de manejo de un vehículo militar con y sin estímulos distractores externos (j1, j2 y j3) para el área occipital (Figura 2a) en los canales O1 y O2 y área temporal (Figura 2b) en los canales T4, T5 y T6. Las líneas muestran la evolución de la Movilidad con el cambio de tipo de EEG, el paso de los estados basales iniciales a las tareas de simulación y vuelta al estado basal con ojos abiertos final. Se observa un incremento del cuantificador al dejar los estados basales e inicial las simulaciones y un decrecimiento del mismo al abandonar la simulación y recuperar el estado basal con ojos abiertos. 
Tabla 2. p-valores del test de hipótesis de comparación por pares de los tipos de EEG en cada canal (F7, F8, O1, O2, P3, P4, T3, T4, T5, T6) para la Movilidad.

\begin{tabular}{|c|c|c|c|c|}
\hline & Basal oc / Tarea j1 & Basal oa / Tarea j1 & Tarea j1 / Tarea j2 & $\begin{array}{c}\text { Basal } \\
\text { oa (pre) / oa(post) }\end{array}$ \\
\hline F7 & 0,003 & 0,000 & 0,243 & 0,890 \\
\hline F8 & 0,001 & 0,000 & 0,089 & 0,720 \\
\hline 01 & 0,000 & 0,000 & 0,894 & 0,067 \\
\hline $\mathbf{O 2}$ & 0,000 & 0,000 & 0,984 & 0,583 \\
\hline P3 & 0,821 & 0,880 & 0,409 & 0,022 \\
\hline P4 & 0,738 & 0,689 & 0,890 & 0,026 \\
\hline T3 & 0,531 & 0,304 & 0,021 & 0,972 \\
\hline T4 & 0,414 & 0,496 & 0,017 & 0,301 \\
\hline T5 & 0,000 & 0,005 & 0,242 & 0,749 \\
\hline T6 & 0,000 & 0,001 & 0,259 & 0,972 \\
\hline
\end{tabular}

T6), con valores más altos durante la tarea. Las diferencias en la zona frontal F7, F8 presentan valores más altos en los EEG con ojos cerrados.

Las diferencias significativas entre la tarea de simulación (j1) y el estado basal con ojos abiertos se dan en F7, F8, T5, T6, O1, O2. En este caso, la Movilidad es mayor en la tarea en áreas occipitales y temporales e inferior en la zona frontal.

No hay diferencias significativas entre las tareas de simulación (j1) y (j3). Entre las simulaciones (j1) y (j2), se observan diferencias al nivel 0.05 en parte del área temporal (T3 y T4).
Las diferencias significativas entre los estados basales con ojos abiertos antes y después de las simulaciones solo ocurren en la zona parietal $(\mathrm{P} 3, \mathrm{P} 4)$.

La figura 6 presenta un gráfico de barras de error con intervalos de confianza (IC) de la media para un nivel de confianza del 95\%, que complementa los resultados obtenidos en el análisis exploratorio con los Box-Plot (canales $\mathrm{O} 1$ y O2). Se observa que existen diferencias significativas, especialmente en el canal O2, ya que en la tarea (j1) el intervalo de confianza no se solapa con los del estado basal (oc) y (oa).
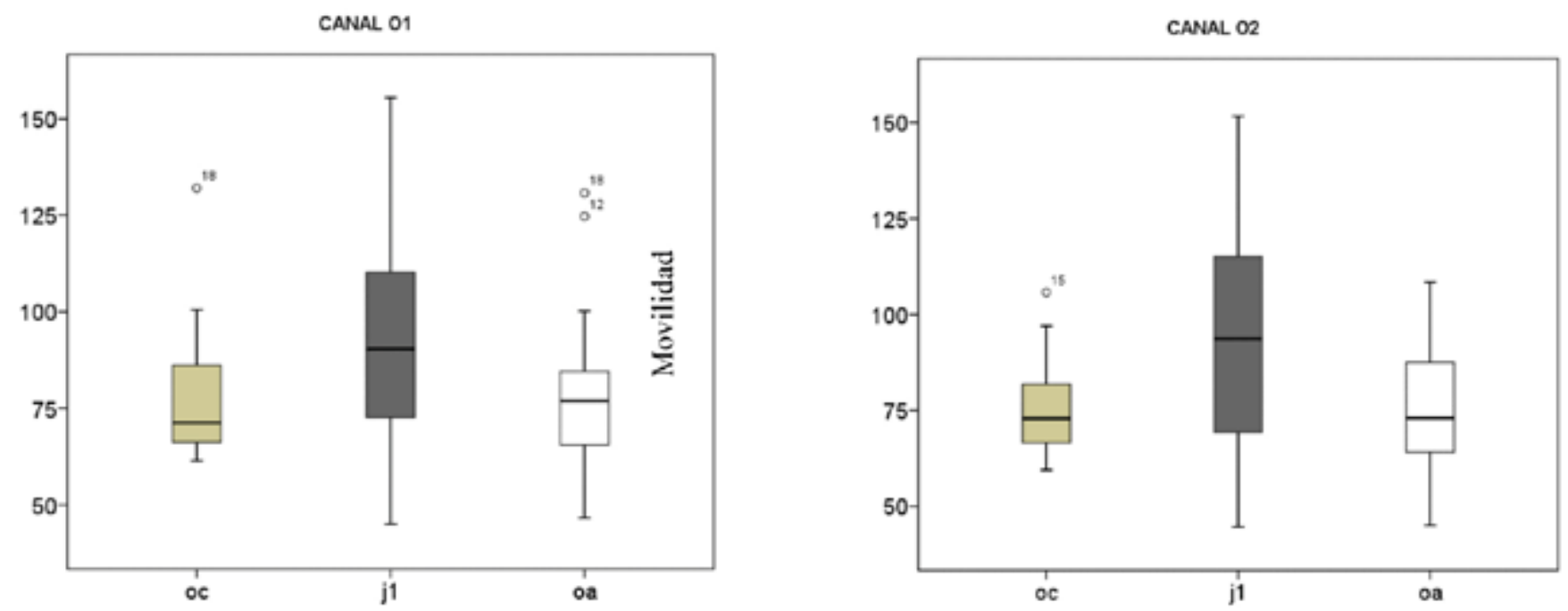

Figura 4. Movilidad por canal del área occipital (O1 y O2) en tres tipos de EEG, basal con ojos cerrados (oc) representado en gris claro, tarea de simulación de manejo de un vehículo militar (j1) en gris oscuro y estado basal con ojos abiertos (oa) en color blanco. Los gráficos Box-Plot representan los valores de la Movilidad obtenidos por los sujetos $(n=36)$ en cada uno de los tres tipos de EEG. Se observa que los valores son más altos y con mayor variabilidad en la tarea de simulación de conducción (j1) que en los estados basales en ambos canales. 

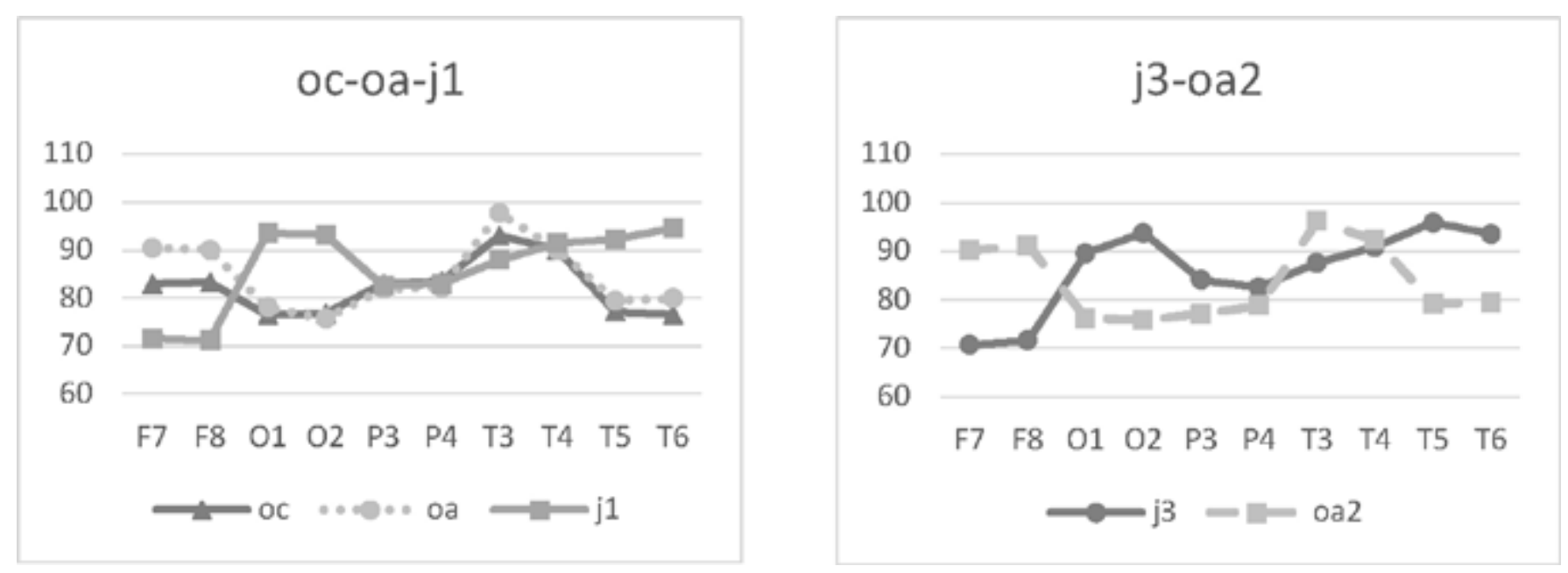

Figura 5. Valores promedio de la Movilidad en cada electrodo durante los estados de reposo y las simulaciones. En la Figura 4 a se comparan los estados basales con ojos cerrados (oc) y abiertos (oa) con la tarea de simulación de manejo de vehículos militares (j1). En la Figura $4 b$ se muestran los valores de la tarea de simulación (j3) y los obtenidos en el estado basal final con ojos abiertos (oa2).
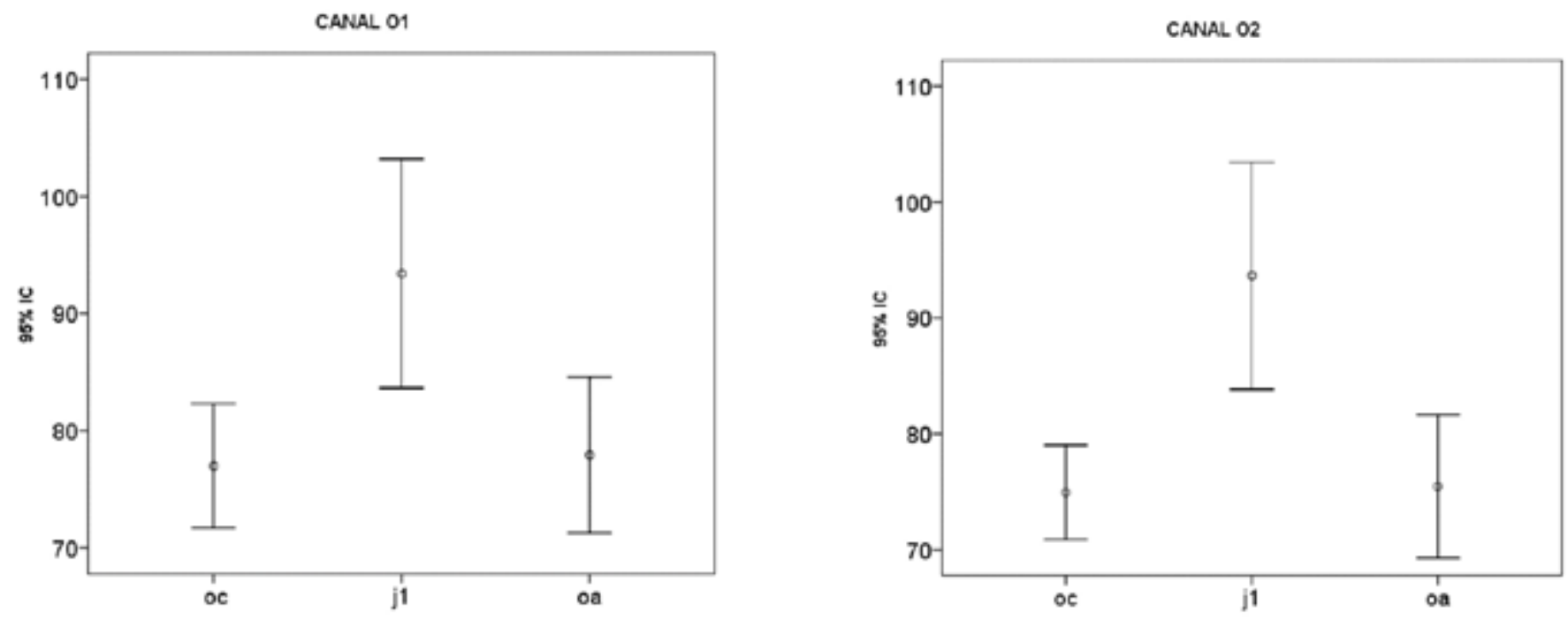

Figura 6. Movilidad por canal del área occipital ( $\mathrm{O} 1$ y $\mathrm{O} 2$ ) en tres tipos de EEG, basal con ojos cerrados (oc), tarea de simulación de manejo de un vehículo militar (j1) y estado basal con ojos abiertos (oa). Los gráficos de error representan la estimación de la media mediante intervalos de confianza al 95\% de nivel de confianza, para cada tipo de EEG. Existen diferencias significativas entre la tarea de simulación de conducción ( $j 1$ ) y los estados basales con ojos cerrados y abiertos ( $p$-valor $<0,05$ ).

Los resultados obtenidos con la dimensión fractal en un trabajo previo corroboran los encontrados en la Movilidad ${ }^{18}$.

\section{DISCUSIÓN}

La conducción militar requiere mayor atención que la civil, ya que el conductor además de atender a las tareas típicas debe observar la complejidad del terreno, la existencia de posibles vehículos enemigos, la monitorización de las comunicaciones y otros posibles estímulos distractores exter- nos. La simulación de la conducción en un simulador puede bajar el nivel de atención, ante la imposibilidad de un accidente real, pero sigue siendo una forma útil de evaluar las variaciones corticales dependiendo del tipo y condiciones de conducción o pilotaje. Así, algunos autores han analizado la actividad cerebral durante las simulaciones de vuelo con diferentes niveles de dificultad. En el trabajo de Astolfi et $a l .{ }^{19}$ se indica un aumento de la densidad de potencia espectral global del EEG en las fases de simulación de despegue y aterrizaje de una aeronave en comparación con las etapas en vuelo simulado. Dussault et al. ${ }^{20}$ detectan estados de ansiedad 
y vigilancia debidos a las variaciones en el esfuerzo mental en diferentes secuencias de simulaciones de vuelo. Otros autores señalan que el espectro de potencia del EEG es sensible a variaciones en la complejidad de los procedimientos de vuelo real ${ }^{21}$. Así, Dussault et al. ${ }^{22}$ detectan un incremento en la actividad de la banda theta durante el vuelo en comparación con los períodos de descanso y una disminución en la banda alfa.

Las relaciones de la actividad cerebral con la sobrecarga mental o la fatiga en tareas de manejo de aeronaves y vehículos son recogidas por Borghini et al. ${ }^{23}$. Se detecta un aumento en la potencia del EEG en la banda theta y una disminución en la banda alfa en aquellas pruebas que requieren una alta carga de trabajo mental. Otros trabajos recientes muestran la existencia de redes cerebrales corticales y subcorticales involucradas en la variación de los espectros de potencia del EEG mientras se realizan tareas de vuelo y conducción ${ }^{20,24}$.

En general, las actividades cerebrales muestran un aumento de la potencia alfa, beta y gamma en las áreas frontal y central en las tareas de atención con una mayor actividad theta, alfa y beta en las tareas de vigilancia. Durante la atención visual, la actividad beta mejora y se observa comúnmente que la sincronización de fase de baja frecuencia del EEG aumenta entre las regiones frontales y parietales del cerebro en tareas que requieren orientación atencional ${ }^{25}$.

Analizando el EEG, Bodala et $a 1 .{ }^{4}$ detectan un aumento en el nivel de vigilancia en una tarea monótona debido a la integración de estímulos visuales ruidosos. Lal \& Craig ${ }^{26}$ y Astolfi et al. ${ }^{19}$ estudian el aumento de la actividad delta a medida que aumenta la fatiga. La supresión de la potencia relativa delta debido a la integración de un estímulo externo está relacionada con la inhibición de la fatiga y el aumento de los niveles de vigilancia.

Nuestro trabajo complementa el estudio de las bandas frecuencias. Los datos obtenidos en el promedio cortical de la Movilidad evidencian cómo este parámetro de cuantificación detecta los cambios de actividad cerebral con el cambio de tipo de EEG.

Las diferencias significativas encontradas en el análisis de varianza confirman que la Movilidad varía en las diferentes áreas cerebrales (canal significativo). También se ha detectado una interacción entre las áreas del cerebro y la tarea realizada por el sujeto (canal-tarea significativo).

Tanto el análisis estadístico descriptivo como el inferencial, revelan un aumento en la Movilidad en las áreas involucradas en las tareas de simulación de manejo de vehículos militares.

La zona frontal refleja un incremento en el cuantificador tras la apertura de ojos en el estado basal y una disminución de dicho parámetro a medida que aumenta la actividad cerebral con una tarea, elevándose además al volver de nuevo al estado basal tras abandonar las tareas de simulación. Por el contrario, en las zonas occipitales y temporales, el parámetro aumenta a medida que la tarea se hace más complicada, es decir, se requiere la activación de áreas cerebrales específicas. Este incremento indica la activación de la red atencional posterior directamente relacionada con la atención visuo-espacial.

Se puede concluir que la Movilidad es un buen indicador de la actividad cerebral, ya que varía en las distintas tareas en las áreas cerebrales involucradas en la realización de las mismas. El aumento considerable de la Movilidad en las zonas occipitales y parte de las temporales, así como las diferencias significativas obtenidas en dichas áreas con el test estadístico muestran la activación del área visual primaria localizada en el lóbulo occipital y la corteza auditiva primaria localizada en el lóbulo temporal.

Los resultados previos obtenidos por nuestro grupo, en trabajos donde se estudiaba la diferencia entre el estado cerebral basal de ojos cerrados y la realización de una tarea, muestran la activación de estas áreas cerebrales ${ }^{27,28}$. Se podría cuestionar si las diferencias halladas en el área visual ponían de manifiesto la activación por la realización de la tarea o simplemente eran consecuencia de la apertura de ojos realizada durante la ejecución de dicha tarea. El trabajo que aquí se presenta puede aclarar esta cuestión, ya que no hay diferencias significativas entre el estado de reposo con los ojos cerrados y los ojos abiertos. Los resultados obtenidos confirman que el desempeño de la tarea es crucial para la activación de frecuencia del cerebro.

Las diferencias encontradas entre la tarea basal ojos abiertos y la tarea de simulación en el área occipital corrobora los resultados anteriores de activación del área visual involucrada en la tarea. Esta activación puede deberse al procesado sensorial que realiza el sujeto durante la tarea atencional. La atención visual es el resultado de una red de conexiones corticales y subcorticales, de descripción prolija, que conforma un circuito cuya entrada es la información captada en la retina y su salida es a través del sistema oculomotor. Los procedimientos descritos resultan de ayuda para una mejor comprensión numérica de las señales electroencefalográficas implicadas en ella.

Este estudio complementa trabajos anteriores donde nuestro grupo analizó las diferencias entre grupos (inter-sujetos). Al comparar la realización de la tarea de simulación de conducción de un vehículo militar (j1) entre un grupo de Caballeros Cadetes y un grupo de Oficiales, se obtuvieron diferencias significativas en las zonas centrales, parietales y temporales ${ }^{18}$. Estudiando las diferencias relacionadas con el género se pudo concluir que ante una misma tarea el grupo de mujeres presentaba valores más altos de activación cerebral que el grupo de hombres ${ }^{29}$. Además, las mujeres son más selectivas a la hora de utilizar las distintas áreas cerebrales. Se sigue trabajando en la realización de pruebas electroencefalográficas durante la simulación y conducción de vehículos militares en el Centro Nacional de Adiestramientro "San Gregorio".

\section{BIBLIOGRAFÍA}

1. Posner MI, Dehaene S. Attentional networks. Trends Neurosci 1994; 17(2): 75-79.

2. Petersen S, Posner M. The attention system of the human brain: 20 years after. Annu Rev Neurosci 2012; 35: 73-89.

3. Colmenero JM, Catena A, Fuentes LJ. Atención visual: Un estudio de las redes atencionales del cerebro. An Psicol 2001; 17(1): 45-67.

4. Bodala IP, Li J, Thakor NV, Al-Nashash H. EEG and eye tracking demonstrate vigilance enhancement with challenge integration. Front Hum Neurosci 2016; 10: 273

5. Friedl, K.E. Military applications of soldier physiological monitoring. J. Sci. Med. Sport 2018, 21, 1147-1153.

6. Chérif, L.; Wood, V.; Marois, A.; Labonté, K.; Vachon, F. Multitasking in the military: Cognitive consequences and potential solutions. Appl. Cogn. Psychol. 2018, 32, 429-439.

7. Kerick, S.E.; Hatfield, B.D.; Allender, L.E. Event-related cortical dynamics of soldiers during shooting as a function of varied task demand. Aviat. Space Environ. Med. 2007, 78, B153-B164. 


\section{Actividad electroencefalográfica durante tareas de simulación de conducción con videojuegos}

8. Blacker, K.J.; Hamilton, J.; Roush, G.; Pettijohn, K.A.; Biggs, A.T. Cognitive training for military application: A review of the literature and practical guide. J. Cogn. Enhanc. 2019, 3, 30-51.

9. Nindl, B.C.; Beals, K.; Witchalls, J.; Friedl, K.E. Military human performance optimization and injury prevention: Strategies for the 21 st century warfighter. J. Sci. Med. Sport 2017, 20, S1-S2.

10. Lu, S.; Zhang, M.Y.; Ersal, T.; Yang, X.J. Workload management in teleoperation of unmanned ground vehicles: Efects of a delay compensation aid on human operators' workload and teleoperation performance. Int. J. Hum. Comput. Int. 2019, 35, 1820-1830.

11. Mao, M.; Xie, F.; Hu, J.; Su, B. Analysis of workload of tank crew under the conditions of informatization. Def. Technol. 2014, 10, 17-21.

12. Micheloyannis S, Vourkas M, Bizas M, Simos P, Stam CJ. Changes in linear and nonlinear EEG measures as a function of task complexity. Brain Topogr 2003; 15: 239-247.

13. Yang H, Wang Y, Wang CJ, Tai HM. Correlation dimensions of EEG changes during mental tasks. Proceedings of the 26th Annual International Conference of the IEEE EMBS San Francisco, CA, USA; 2004: 616-619.

14. Navascués MA, Sebastián MV. Fitting curves by fractal interpolation: An application to the quantification of cognitive brain processes. In: Thinking in Patterns: Fractals and Related Phenomena in Nature. Novak MM (ed.) World Scientific Publishers; 2004: 143-154.

15. Navascués MA, Sebastián MV. Time domain indices and discrete power spectrum in electroencephalographic processing. Int J Comp Math 2009; 86(1011): 1968-1978.

16. Hjorth B. EEG analysis based on time domain properties. Electroenceph and Clin Neurophys 1970; 29: 306-312.

17. Navascués MA, Sebastián MV, Ruiz C, Iso JM. A numerical power spectrum for electroencephalographic processing. Math Method Appl Sci 2016; 39(16): 4680-4687.

18. Idiazábal MA, Sebastián MV, Navascués MA, Arcos C, Arana MV, Ruiz C, et al. A cortical study of the attention in military simulation test. Rev Neurol 2018; 66(10): 331-339.

19. Astolfi L, Toppi J, Borghini G, Vecchiato G, Isabella R, De Vico Fallani F, et al. Study of the functional hyperconnectivity between couples of pilots during flight simulation: An EEG hyperscanning study. Proceedings of the Annual International Conf Proc IEEE Eng Med Biol Soc; 2011: 2338-2341.

20. Dussault C, Jouanin JC, Philippe M, Guezennec CY. EEG and ECG changes during simulator operation reflect mental workload and vigilance. Aviat Space Environ Med 2005; 76(4): 344-351.

21. Di Stasi LL, Díaz-Piedra C, Suárez J, McCamy MB, Martínez-Conde S, Roca-Dorda J, et al. Task complexity modulates pilot electroencephalographic activity during real flights. Psychophysiology 2015; 52: 951-956.

22. Dussault C, Jouanin JC, Guezennec CY. EEG and ECG changes during selected flight sequences. Aviat Space Environ Med 2004; 75(10): 889-897.

23. Borghini G, Astolfi L, Vecchiato G, Mattia D, Babiloni F. Measuring neurophysiological signals in aircraft pilots and car drivers for the assessment of mental workload, fatigue and drowsiness. Neurosci Biobehav Rev 2014; 44: $58-75$.

24. Borghini G, Vecchiato G, Toppi J, Astolfi L, Magkione A, Isabella R, et al. Assessment of mental fatigue during car driving by using high resolution EEG activity and neurophysiologic indices. Conf Proc IEEE Eng Med Biol Soc; 2012. p. 6442-6445.

25. Dombrowe I, Hilgetag CC. Occipitoparietal alpha-band responses to the graded allocation of top-down spatial attention. J Neurophysiol 2014; 112: 1307-1316.

26. Lal, SK, Craig A. A critical review of the psychophysiology of driver fatigue. Biol Psychol 2001; 55: 173-194.

27. Sebastián MV, Navascués MA, Ruiz C, Iso JM, Arcos C, Arana V, et al. Estudio electroencefalográfico de la atención durante la realización de tareas de simulación militar. Dena Arto et al. Actas: II Congreso Nacional de $\mathrm{i}+\mathrm{d}$ en Defensa y Seguridad, DESEi+d 2014. Ed. Centro Universitario de la Defensa; 2014. p. 487-494.

28. Sebastián MV, Navascués MA, Valdizán JR. Cuantificación de señales electroencefalográficas para el estudio de la atención. González-Marcos, A.P. (coord.) Actas: Congreso Nacional de I+D en Defensa y Seguridad, DESEi+d 2013., Madrid, 2013. p. 543-554.

29. Sebastián MV, Arana MV, Arcos C, Navascués MA, Idiazábal MA, Ruiz $\mathrm{C}$, et al. Attention processes in military simulation tasks: Gender-associated differences. Rev Cient Soc Esp Enferm Neurol 2018; 48: 2-8. 